\title{
La Schistosomose intestinale dans les forêts marécageuses à Pterocarpus de Guadeloupe (Antilles françaiscs)
}

\author{
Ecologie du mollusque vecteur, Biomphalaria glabrata \\ et de son parasite Scbistosoma mansoni \\ par J.-P. POINTIER * et A. THERON ** \\ * Laboratoire de Biologie Marine et Malacologie (Directeur: $P^{r}$ B. Salvat), \\ Ecole Pratique des Hautes Etudes, 55, rue de Buffon, F 75005 Paris. \\ ** Département de Biologie Animale (Directeur : Pr C. Combes), \\ Université, avenue de Villeneuve, F 66025 Perpignan Cedex.
}

\section{Résumé.}

Dans la mangrove lacustre de Dubelloy-Devarieux (Guadeloupe), la dynamique des populations de $B$. glabrata et de la transmission de $S$. mansoni est étroitement dépendante de l'alternance saison sèche, saison des pluies. La remise en eau de la mangrove (septembre) permet chaque année un renouvellement des peuplements malacologiques. L'analyse des classes de taille montre que le cycle de développement d'une population de B. glabrata comporte deux saisons des pluies séparées par une saison sèche. La prévalence d'infestation des Mollusques $(0,25 \%)$ et les densités de cercaires de $S$. mansoni $(0,8 \mathrm{c} / \mathrm{I})$ sont faibles. Le rythme de présence des cercaires en milieu stagnant montre une acrophase à 14 heures. L'homme et le rat sont parasités. La contamination des populations murines passe de $20 \%$ à la remise en eau, à près de $100 \%$ en fin de saison des pluies. Les facteurs pouvant favoriser l'infestation des rats sont discutés.

\section{Summary.}

Intestinal schistosomiasis in the freshwater mangrove of Guadalupe (French Antilles). Ecology of the vector, Biomphalaria glabrata and its parasite Schistosoma mansoni.

In the freshwater mangrove of Dubelloy-Devarieux (Guadalupe), the dynamics of populations of $B$. glabrata and the transmission of $S$. mansoni, mostly depends on the alternation

Accepté le 18 décembre 1978. 
of dry and rainy season. The flooding of the mangrove (in september), permits a yearly renewal of the malacological populations. The sizes analysis of the snails shows that the life cycle of $B$. glabrata include two rainy seasons separated by one dry season. The prevalence of $B$. glabrata $(0,25 \%)$ and cercariae densities $(0,8 \mathrm{c} / \mathrm{l})$ are very slow. The acrophase of the rythm of presence in standing waters is reached at $2 \mathrm{pm}$. Men and rats are infected. The infections of rats is of $20 \%$ at the beginning of the rainy season and of $100 \%$ at the end of the rainy season. Principal factors involved in the infection of rats are discussed.

\section{Introduction}

Les forêts à Pterocarpus officinalis constituent en Guadeloupe une formation végétale très particulière qui fait suite à la mangrove marine typique et le terme «mangrove lacustre » a été utilisé pour leur dénomination par Lasserre (1961). Au début de la saison des pluies, en août-septembre, les bois sont envahis par les eaux douces, puis s'assèchent pendant le carême vers le mois de mars. Des études préliminaires nous avaient montré la présence dans la mangrove de Dubelloy-Devarieux d'une importante population de Biomphalaria glabrata, Mollusque vecteur de la Schistosomose intestinale (Pointier, 1974-1976 ; Pointier et Combes, 1976 ; Pointier et al., 1977). Par la suite, des prospections intensives ont confirmé la présence de $B$. glabrata dans presque toutes les zones à Pterocarpus des deux îles guadeloupéennes (fig. 1).

En 1975 (1), Nassi et al. signalent la présence de quelques rats intensément parasités par Schistosoma mansoni dans la mangrove de Dubelloy-Devarieux, et en 1977 Mougeot et Golvan découvrent des rats parasités dans de nombreuses mangroves lacustres de Guadeloupe. Par ailleurs, une étude menée de 1976 à 1978 dans la zone de Dubelloy-Devarieux a démontré l'intensité de la parasitose au sein des populations murines dont la prévalence passe de $20 \%$ au moment de la remise en eau du milieu à près de $100 \%$ en fin de saison des pluies (Combes et Delattre, 1978).

En 1978, dans la même localité, une enquête menée par Rioux et al. (en préparation) révèle la présence de la maladie chez l'homme (17\% de la population du village voisin de Dubelloy sont porteurs d'anticorps).

Parallèlement à ces enquêtes menées sur les populations humaines et murines, l'étude du Mollusque vecteur et de son parasite a été entreprise lors de la remise en eau de la mangrove de Dubelloy-Devarieux en septembre 1977 et s'est poursuivie jusqu'à son assèchement en mars 1978.

\section{Méthodes}

La zone étudiée, la mangrove de Dubelloy-Devarieux, est située à l'ouest de la Grande-Terre entre Vieux Bourg et Morne à l'Eau (fig. 1 et 2), et comprend trois zones

(1) Action concertée de la Délégation Générale à la Recherche Scientifique et Technique. Lutte biologique : Vecteurs. Coordonnateur: Pr. Y.J. Golvan. La partie terrain de ce travail a été réalisée à la Station de Zoologie de l'Institut National de la Recherche Agronomique, Petit-Bourg (Guadeloupe). 




Fig. 1. Distribution de Biomphalaria glabrata dans la mangrove lacustre de Guadeloupe. 1: Mangrove de Montplaisir. 2: Mangrove de Morne Rouge. 3: Mangrove à Espérance. 4: Mangrove de la Rivière Houaromond. 3: Mangrove à Destrellan. 6: Mangrove entre le Raizet et la Gabarre. 7: Mangrove de Belle Plaine. 8: Mangrove de Perrin Nord. 9: Mangrove de Vieux Bourg. 10: Mangrove de Dubelloy-Devarieux. 11: Mangrove des Rotours. 12: Mangrove de Roujol. 13: Mangrove de Port-Louis. 14: Mangrove de Gosier. 




Fig. 2. Mangrove lacustre de Dubelloy-Devarieux. Situation et topographie de la zone étudiée. Position du transect d'échantillonnage (carrés 1 à 14) et des 11 zones complémentaires de récolte (en pointillé). 
principales caractérisées par la végétation : une prairie à Graminées, une zone de cultures et les bois proprements dits.

\section{Echantillonnage des Mollusques.}

L'échantillonnage des Mollusques a été réalisé le long d'un transect traversant les trois principales strates végétales qui sont inondées à la saison des pluies: prairie à Graminées, cultures de Colocasia esculenta et bois à Pterocarpus officinalis. La méthode consiste à récolter les Mollusques à l'aide d'une passoire dans des carrés de $10 \mathrm{~m}$ de côté s'échelonnant le long du transect. La récolte est toujours effectuée par le même opérateur, sa durée est limitée à $1 / 4$ d'heure. Le nombre de carrés s'échelonnant le long du transect est de 14 (fig. 2). Les prélèvements sont effectués toutes les deux semaines, les résultats sont exprimés en Mollusques/quart d'heure. Cette méthode ne nous permet d'obtenir qu'une certaine fraction de la population réellement présente car il est impossible d'effectuer des prélèvements exhaustifs dans de tels milieux. La méthode étant toujours la même pour chaque prélèvement, les résultats donnent une image relative de la répartition des $B$. Glabrata dans les différents secteurs du transect. Cependant, par la méthode de marquage-recapture nous avons pu estimer qu'un Mollusque/quart d'heure représentait environ 8,8 Mollusques $/ \mathrm{m}^{2}$. Les résultats ne concernent que les Mollusques de taille supérieure à 5-6 mm car il est impossible de récolter les nouveau-nés $(<1 \mathrm{~mm})$ et les juvéniles sont toujours très sous-estimés (Dazo et al., 1966).

\section{Estimation de la croissance des Mollusques.}

La croissance des Mollusques a été estimée par une méthode d'élevage in situ dans des cages (Levèque et Pointier, 1976). Cette méthode qui utilise l'équation de Von Bertalanffy nous a permis de calculer deux courbes de croissance, une en septembre 1977 peu après la remise en eau, et l'autre en novembre lorsque la nouvelle génération de Mollusques est apparue. Ces deux courbes ont été comparées avec l'évolution des deux principales cohortes de Mollusques.

\section{Estimation de la prévalence des Mollusques parasités.}

De part et d'autre du transect, 11 zones de récoltes complémentaires ont été définies pour estimer la prévalence et préciser la localisation des Mollusques parasités. La détection de la parasitose est effectuée par le test de sortie des cercaires sur chacun des Mollusques récoltés.

\section{Mesure des densités cercariennes de Schistosoma mansoni.}

Les mesures des densités cercariennes de $S$. mansoni ont été faites en utilisant la technique de filtration différentielle (Théron, 1979). Les échantillons d'eau de 10 litres sont prélevés dans les zones d'échantillonnage des Mollusques, formolés (10 cc de formol par prélèvement) et analysés au laboratoire. Pour la détermination du rythme 
de présence, les prélèvements sont effectués autour d'une source d'émission cercarienne expérimentale toutes les heures pendant un cycle journalier.

\section{Résultats}

\section{Répartion de Biomphalaria glabrata dans la mangrove.}

Une précédente étude (Pointier et Combes, 1976) a montré que les populations de $B$. glabrata étaient particulièrement denses à la lisière des bois de Pterocarpus et disparaissaient très rapidement sous les arbres. Cette distribution a également été observée le long du transect en 1977. Dès la remise en eau les populations de B. glabrata sont concentrées essentiellement dans la zone de lisière correspondant aux carrés 11 et 12 (fig. 2 et 3 ). La remise en eau de la mangrove commence en septembre par les bois et la zone de lisière, puis la nappe envahit les cultures de Colocasia et la prairie n'est inondée que fin octobre (fig. 3). Le retrait des eaux

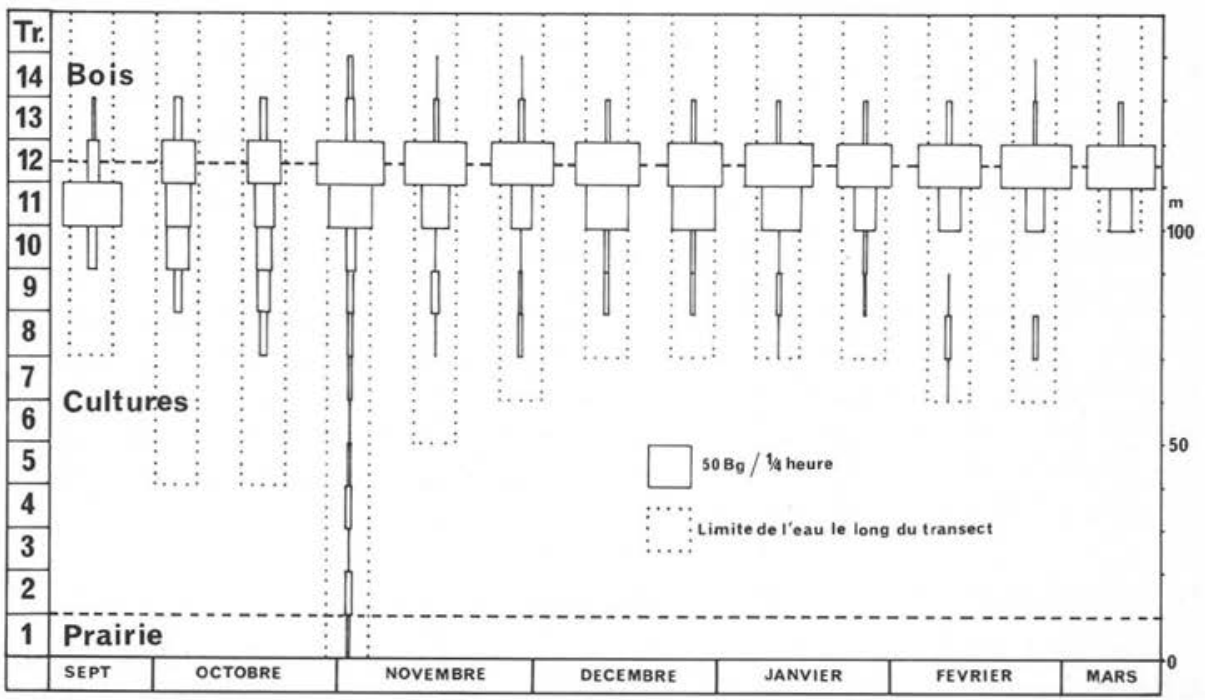

Fig. 3. Mangrove lacustre de Dubelloy-Devarieux. Distribution et évolution des populations de Biomphalaria glabrata le long du transect prairie-bois au cours de la saison des pluies 1977-1978.

est ensuite très rapide, puis à partir de décembre la nappe se stabilise jusqu'en mars vers la zone médiane des cultures de Colocasia. Enfin, la première quinzaine de mars voit la disparition rapide des dernières nappes d'eau. L'assèchement complet est réalisé vers le 20 mars 1978. Durant ces 6 mois, la plus grande partie des populations de B. glabrata est restée cantonnée dans une zone étroite correspondant à la lisière des bois. 


\section{Evolution des populations de Biomphalaria glabrata.}

L'analyse des classes de taille des Mollusques nous permet de suivre l'évolution de deux cohortes bien distinctes de septembre 1977 à mars 1978 (fig. 4). La première cohorte qui correspond aux Mollusques ayant résisté à 6 mois d'assèchement est constitué par des individus adultes de taille moyenne de $15 \mathrm{~mm}$ et qui vont reprendre leur croissance dès la remise en eau de septembre. La courbe de croissance calculée à l'aide de l'équation de Von Bertalanffy correspond bien à l'évolution de la cohorte qui se stabilisera ensuite dès le mois de novembre (fig. 4, courbe 1). On retrouvera cette cohorte en mars 1978 .

La deuxième cohorte qui correspond à la nouvelle génération de Mollusques, apparaît dans les prélèvements vers la mi-octobre. La croissance des Mollusques est rapide fin octobre et la courbe calculée par l'équation de Von Bertalanffy correspond bien à l'évolution de la cohorte de jeunes pendant cette période (fig. 4, courbe 2). Cependant dès la fin novembre, la croissance ralentit puis cesse complèment (fig. 4 , courbe 3). Cette stagnation de la croissance qui dure environ 2 mois (fin novembre à fin janvier) est problablement due aux températures assez basses qui sont observées

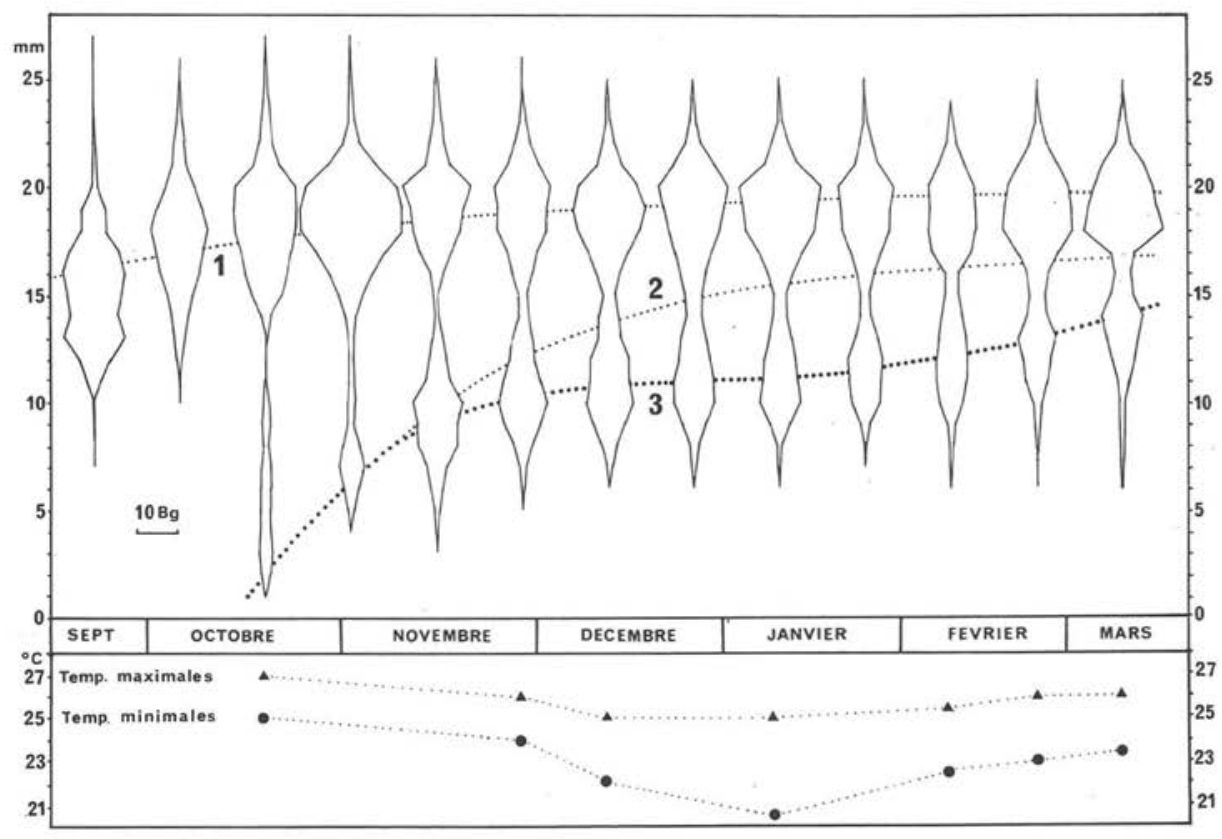

Fig. 4. Mangrove lacustre de Dubelloy-Devarieux. Evolution des classes de taille et croissance de Biomphalaria glabrata au cours de la saison des pluies 1977-1978. 1: courbe de croissance calculée à l'aide de l'équation de Von Bertalanffy en octobre 1977. 2: courbe de croissance calculée à l'aide de l'équation de Von Bertalanffy en novembre 1977. 3: évolution de la cohorte de jeunes au cours de la saison des pluies. 
à cette période qui est la plus fraîche de l'année. Les températures minimales des eaux sont en effet de $20-21^{\circ} \mathrm{C}$ et les températures maximales de $24-25^{\circ} \mathrm{C}$, alors qu'en octobre les minima étaient de l'ordre de $24-25^{\circ} \mathrm{C}$ et les maxima de $26-27^{\circ} \mathrm{C}$. Ceci est confirmé par la reprise de croissance en février où les températures sont nettement en hausse : $22-23^{\circ} \mathrm{C}$ pour les minima et $25-26^{\circ} \mathrm{C}$ pour les maxima.

Ainsi, au seuil de la saison sèche, on peut distinguer très nettement, par la structure en taille, deux cohortes différentes de Mollusques : une première cohorte qui a estivé durant 6 mois puis qui termine son cycle de développement durant les 6 mois suivants, et une deuxième cohorte issue de la première, qui a commencé son cycle de développement un mois après la remise en eau et qui ne le terminera qu'à la saison des pluies suivante. Le cycle de développement normal d'une population de B. glabrata dans la mangrove lacustre comporte donc deux saisons des pluies (environ deux fois 6 mois) séparées par une saison sèche (environ une fois 6 mois) (2).

\section{Prévalence d'infestation des $B$. glabrata par $S$. mansoni.}

Nous avons étudié l'évolution du parasitisme des B. glabrata de la mangrove au cours de la saison des pluies entre le 15 septembre 1977 et le 15 mars 1978 (tableau I). Les prélèvements, effectués tous les 15 jours, représentent un total de 10747 Mollusques. Seuls 26 B. glabrata se sont révélés positifs au test d'émission des cercaires de $S$. mansoni, soit une prévalence globale moyenne de $0,25 \%$, cette prévalence est extrêmement faible. Il n'existe pas de fluctuation significative du parasitisme des Mollusques au cours de la saison des pluies.

Tableau I. - Variation du parasitisme de B. glabrata au cours de la saison des pluies.

\begin{tabular}{|l|c|c|c|c|c|c|c|c|c|c|c|c|c|c|}
\hline $\begin{array}{l}\text { Dates des prélève- } \\
\text { ments ............... }\end{array}$ & $\begin{array}{c}09- \\
77\end{array}$ & $\begin{array}{l}10- \\
77\end{array}$ & $\begin{array}{l}10- \\
77\end{array}$ & $\begin{array}{c}11- \\
77\end{array}$ & $\begin{array}{l}11- \\
77\end{array}$ & $\begin{array}{c}11- \\
77\end{array}$ & $\begin{array}{c}12- \\
77\end{array}$ & $\begin{array}{c}12- \\
77\end{array}$ & $\begin{array}{c}01- \\
78\end{array}$ & $\begin{array}{c}01- \\
78\end{array}$ & $\begin{array}{c}02- \\
78\end{array}$ & $\begin{array}{c}02- \\
78\end{array}$ & $\begin{array}{c}03- \\
78\end{array}$ & Total \\
\hline B. glabrata récoltés . & 597 & 619 & 677 & 954 & 617 & 925 & 628 & 936 & 1155 & 973 & 899 & 1002 & 965 & 10747 \\
\hline B. glabrata positifs .. & 0 & 3 & 3 & 4 & 3 & 2 & 2 & 0 & 1 & 3 & 1 & 1 & 3 & 26 \\
\hline Prévalence $\% \ldots \ldots \ldots$ & 0 & 0,48 & 0,44 & 0,42 & 0,49 & 0,22 & 0,32 & 0 & 0,09 & 0,31 & 0,11 & 0,10 & 0,31 & $\begin{array}{c}\text { Moyenne } \\
0,25 \%\end{array}$ \\
\hline
\end{tabular}

Les Mollusques parasités sont essentiellement localisés dans la zone de lisière (fig. 2) qui héberge la majorité de la population de B. glabrata.

\section{Rythme de présence et densités cercariennes de $S$. mansoni.}

Avant de mesurer les densités réelles des cercaires de $S$. mansoni dans la mangrove, nous avons cherché à déterminer leur rythme de présence. Si celui-ci est maintenant

(2) Les Mollusques ayant survécu à la saison sèche sont facilement reconnaissables à leur coquille. Celle-ci porte la trace de la reprise de croissance qui s'effectue lors de la remise en eau de la mangrove. 
bien connu en milieu courant (Théron et al., 1977-1978), les données concernant les milieux stagnants sont encore insuffisantes.

a) Rythme de présence cercarienne en mangrove:

La très faible prévalence d'infestation des Mollusques de la mangrove (§ 3) ne nous a pas permis de déterminer ce rythme dans des conditions entièrement naturelles.

Pour pallier l'insuffisance des Mollusques infestés, nous avons placé des Mollusques émetteurs de cercaires dans une cage elle-même immergée dans la mangrove. Nous avons ainsi effectué 10 cycles de filtrations horaires en milieu stagnant. Les résultats résumés dans le tableau II sont exprimés en cercaires par litre et en \% du total journalier des densités horaires. Cette conversion permet d'établir une seule courbe représentative de l'évolution circadienne des densités cercariennes de $S$. mansoni en milieu stagnant (fig. 5). Les caractéristiques de cette évolution sont les suivantes :

- acrophase de présence à $14 \mathrm{~h}$ (heure légale très voisine de l'heure solaire en Guadeloupe),

- densités encore positives après 19 heures.

Fig. 5. Mangrove lacustre de Dubelloy-Devarieux. Rythme de présence des cercaires de Schistosoma mansoni en milieu stagnant. Les densités cercariennes sont exprimées en pourcentage de leur somme journalière.

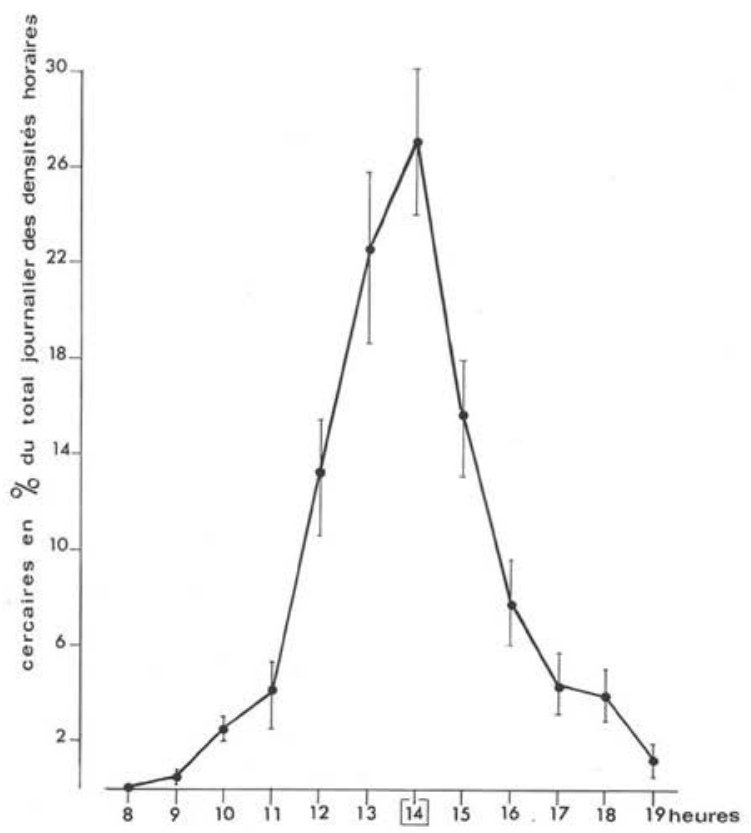

Il est intéressant de remarquer que la position de l'acrophase est décalée de +3 heures par rapport à celle observée en milieu courant $(11 \mathrm{~h})$ (Théron et al., 
Tableau II. - Variations journalières des densités horaires de cercaires de Schistosoma mansoni en milieu stagnant (mangrove lacustre). Les densités sont exprimées en carcaires par litre (c/l), et en \% de leur somme journalière ( $d h)$.



1977). Ce décalage s'explique par une dispersion des cercaires moins importante en l'absence de courant (Négron-Aponte et Jobin, 1977).

Il faut cependant noter qu'il s'agit ici du décalage minimum de l'acrophase correspondant à une source unique d'émission de cercaires. En effet, l'émission de cercaires par des sources multiples, suffisamment proches, peut compenser dans une 
certaine mesure la dilution du nuage cercarien des sources ponctuelles. Ceci se traduirait alors par un décalage plus important de l'acrophase, augmentant encore les densités cercariennes du début de la nuit.

Nous avons analysé des échantillons d'eau prélevés dans la mangrove à différentes heures de la journée. Dans tous les cas, les densités maximales sont observées à $14 \mathrm{~h}$. Au cours de ces expériences, nous avons pu remarquer que les guppies (Poecilia reticulata) étaient souvent attirés vers la source d'émission de cercaires. Bien que les densités de cercaires soient très faibles dans la nature, l'impact de la prédation des cercaires par ces poissons ne saurait être totalement écarté. D'autant plus qu'au maximum d'émission, il se produit autour du Mollusque une augmentation brutale du nombre de cercaires de nature à provoquer un phénomène de «switching».

b) Densités cercariennes de S. mansoni en Mangrove:

Nous avons mesuré les densités des cercaires de $S$. mansoni en mangrove à quatre reprises au cours du mois d'octobre 1977. Les prélèvements ont été effectués à $14 \mathrm{~h}$ dans les zones (1 à 10) d'échantillonnage des Mollusques. Ces densités sont extrêmement faibles, le maximum observé est de $0,8 \mathrm{c} / 1$ à 14 heures. Elles reflètent parfaitement la faible prévalence $(0,46 \%)$ d'infestation des Mollusques détectée au mois d'octobre. Seuls les prélèvements effectués dans les zones 4 et 5 ont été positifs (tableau III). Dans les autres zones, le petit nombre de B. glabrata infestés ne nous a pas permis de détecter des cercaires. Nous pensons cependant que ces densités sont du même ordre de grandeur que celles mesurées en zone 5.

Tableau III. - Densités cercariennes de S. mansoni en c/l à 14 heures mesurées au mois d'oclobre dans les zones d'échantillonnage des Mollusques.

\begin{tabular}{|c|c|c|c|c|c|c|c|c|c|c|}
\hline Zones d'échantillonnage & 1 & 2 & 3 & 4 & 5 & 6 & 7 & 8 & 9 & 10 \\
\hline Densités ...... & - & - & - & 0,1 & 0,7 & - & - & - & - & - \\
\hline Cercariennes & - & - & - & - & 0,8 & - & - & - & - & - \\
\hline$(\mathrm{c} / 1) \quad \ldots \ldots \ldots \ldots \ldots \ldots$ & - & - & - & - & - & - & - & - & - & - \\
\hline A 14 heures $\ldots$.. & - & - & - & - & 0.7 & - & - & - & - & - \\
\hline
\end{tabular}

\section{Discussion et conclusions}

Dans la mangrove lacustre de Guadeloupe, la transmission de la Schistosomose intestinale apparaît étroitement dépendante des conditions climatiques et en particulier de l'alternance saison sèche, saison des pluies.

Le Mollusque vecteur, Biomphalaria glabrata, est présent dans les trois zones prospectées, prairie à Graminées, cultures de Colocasia et bois à Pterocarpus. Cependant, la plus grande partie de ses populations reste cantonnée à la lisière des bois qui semble constituer la zone la plus favorable. Le renouvellement des peuplements mala- 
cologiques est assuré chaque année après la remise en eau d'automne, et l'analyse des classes de taille des Mollusques permet de distinguer deux cohortes d'importance sensiblement égale: l'une correspond aux Mollusques ayant estivé durant les quelques mois de la saison sèche précédente et l'autre correspondant à la nouvelle génération. Les densités de Mollusques restent stables pendant toute la durée de la saison des pluies. La mortalité provoquée par la saison sèche (Pointier et Combes, 1976) et qui atteint plus particulièrement les Mollusques, ayant déjà subit une estivation est compensée chaque année par une arrivée massive de jeunes dès la remise en eau de la mangrove.

La prévalence d'infestation à $S$. mansoni des Mollusques est très faible durant toute la saison des pluies. Les premiers Mollusques parasités ont été mis en évidence en octobre, plus d'un mois après la remise en eau. Il est donc probable que ces Mollusques aient été infestés tout au début de la saison des pluies. Cependant il n'est pas à exclure que quelques individus hébergeant des formes immatures de $S$. mansoni aient pu survivre durant la saison sèche et continuer le développement de leur parasitose lors de la remise en eau. Ce phénomène a en effet été observé par plusieurs auteurs (Barbosa et Olivier, 1958 ; Richards, 1967).

Les populations murines (essentiellement Rattus rattus) présentes dans la zone étudiée ont fait l'objet d'une enquête parasitologique de 1976 à 1978 (Combes et Delattre, 1978 ; Mougeot et al., 1978). Chez le rat, la prévalence d'infestation passe de $20 \%$ au moment de la remise en eau de la mangrove (septembre) à près de $100 \%$ en fin de saison des pluies (mars) et cela, malgré une population de Mollusques faiblement contaminée $(0,25 \%)$ et des densités cercariennes très basses $(0,8 \mathrm{c} / 1)$. Ces résultats sont inversés par rapport à ceux observés au cours d'une enquête similaire réalisée en milieu courant (Rioux et al., 1977 ; Théron et al., 1978). Dans les canaux, nous avons évalué à $12 \%$ la prévalence globale d'infestation des $\mathrm{B}$. glabrata et entre 50 et 250 cercaires/litre les densités cercariennes, alors que seulement $40 \%$ des rats sont parasités.

La comparaison de ces deux situations épidémiologiques fait apparaître qu'en milieu stagnant les populations murines sont fortement parasitées alors que la prévalence d'infestation des Mollusques est 50 fois plus faible et les densités de cercaires 100 fois inférieures à celle d'un site de transmission courant. Il faut donc admettre qu'il existe en milieu stagnant un certain nombre de facteurs favorisant l'infestation des rats. Parmi ces facteurs, le comportement des cercaires d'une part, et plus particulièrement le rythme de présence cercarienne, nous paraît jouer un rôle prépondérant dans la réussite de l'infestation du Vertébré. En effet, en milieu stagnant, une acrophase de présence retardée et des densités cercariennes encore positives au début de la nuit sont de nature à favoriser l'infestation d'animaux à mœurs crépusculaires. L'absence de courant contribue également à augmenter l'infectivité des cercaires (Radke et al., 1961 ; Webbe, 1966). D'autre part, le comportement des rats vis-à-vis de l'eau est un facteur essentiel de la contamination (Golvan et Combes, 1978). Ce dernier reste à préciser, mais on peut penser que pendant toute la saison des pluies, les populations murines de la mangrove ont des contacts avec l'eau plus fréquents que les populations qui occupent le bord des canaux dont les variations de débit sont de faible amplitude. 
Au niveau humain, une enquête réalisée par Rioux et al. (en préparation) sur la quasi-totalité des habitants du village de Dubelloy (146 personnes) a montré que, sur 46 personnes fréquentant la Mangrove, 18 étaient porteurs d'anticorps et 9 présentaient des œufs de $S$. mansoni dans leurs selles. Ceci montre d'une part que l'homme se contamine en mangrove et d'autre part qu'il est probablement lui-même contaminant pour le Mollusque.

Alors qu'il nous a été possible de démontrer le rôle prépondérant que joue l'homme par rapport au rat dans le fonctionnement d'un site de transmission courant, il est difficile, dans l'état actuel de nos connaissances, de dire s'il existe en mangrove une préséance de l'un ou l'autre hôte dans le fonctionnement du foyer. La réponse dépend de plusieurs facteurs, parmi lesquels la quantité d'œufs et de miracidiums infestants produits par les rats et les comportements respectifs de l'homme et du rat vis-à-vis de l'eau.

\section{REMERCIEMENTS}

Nous remercions $\mathrm{M}$. le $\mathrm{P}^{r}$ J.-A. Rioux et $\mathrm{M}^{\mathrm{me}}$ le $\mathrm{D}^{r} \mathrm{G}$. Lanotte (Faculté de Médecine de Montpellier) qui ont bien voulu nous communiquer les résultats de leur enquête sur l'infestation humaine dans le secteur de Dubelloy-Devarieux.

Nous remercions P. Delattre (I.N.R.A.) qui a bien voulu nous communiquer les résultats concernant les populations murines de la Mangrove.

\section{Bibliographie}

Barbosa (F.S.), Olivier (L.), 1958: Studies on the snail vectors of Bilharziasis mansoni in North eastern Brazil. Bull. Wld. Hlth. Org., 18, 895-908.

Combes (C.), Delattre (P.), 1978 : Recherches sur l'infestation de Rattus rattus et Rattus norvegicus par Schistosoma mansoni dans un foyer des Antilles. Fourth International Congress of Parasitology, Warszawa, C 99-100.

Dazo (B.C.), Hairston (N.G.), Dawood (I.K.), 1966: The ecology of Bulinus truncatus and Biomphalaria alexandrina and its implication for the control of bilharziasis in the Egypt, 49 project area. Bull. Wld. Hlth. Org., 35, 339-356.

Golvan (Y.J.), Combes (C.), 1978: Sur la responsabilité des rats sauvages dans l'entretien des foyers de Schistosomose intestinale. Fourth International Congress of Parasitology, Warszawa, C 98.

Lasserre (G.), 1961: La Guadeloupe. Etude géographique. Thèse de doctorat. Union française d'impression. 259 p.

Lévèque (C.), Pointier (J.P.), 1976 : Study of the growth of Biomphalaria glabrata and other planorbidae in Guadeloupe (West Indies). Ann. Trop. Med. Parasitol., 70, 199-204.

Mougeot (G.), Golvan (Y.J.), 1977: Aspects de la Bilharziose murine dans la Mangrove douce et l'arrière Mangrove de la Grande-Terre en Guadeloupe. Ann. Parasitol. Hum. Comp., 52, 623-628.

Mougeot (G.), Golvan (Y.J.), Poirot (J.L.), Marteau (M.), Delattre (P.), 1978: Enquête séroimmunologique par immunofluorescence de la Bilharziose murine en Guadeloupe. Intérêt épidémiologique, étude comparative dans différents biotopes. Ann. Parasitol. Hum. Comp., 3, 271-284.

Nassi (H.), Lancastre (F.), Poirot (J.L.), 1975 : Rat et Bilharziose en Guadeloupe. Nouvelles données. Ann. Parasitol. Hum. Comp., 50, 731-733. 
Negron-Aponte (H.), Jobin (W.R.), 1977 : Guidelines for spacing and timing of samples to detect populations of Schistosoma mansoni cercariae in the field. Int. J. Parasitol., 7, 123-126.

Pointier (J.P.), 1974: Faune malacologique dulçaquicole de l'île de la Guadeloupe (Antilles françaises). Bull. Mus. Hist. Nat. Paris, 159, 905-933.

Pointier (J.P.), 1976: Répartition locale et biogéographie des Mollusques dulçaquicoles de l'île de la Guadeloupe (Antilles françaises). Mal. Rev., 85-103.

Pointier (J.P.), Combes (C.), 1976: La saison sèche en Guadeloupe (Antilles françaises) et ses conséquences sur la démographie des Mollusques dans les biotopes à Biomphalaria glabrata (Say, 1818) vecteur de la Bilharziose intestinale. Terre Vie, 30, 121-147.

Pointier (J.P.), Salvat (B.), Golvan (Y.J.), Delplanque (A.), 1977 : Principaux facteurs régissant la densité des populations de Biomphalaria glabrata (Say, 1818), Mollusque vecteur de la Schistosomose en Guadeloupe (Antilles françaises). Ann. Parasitol. Hum. Comp., 52, 277-323.

Radke (H.G.), Ritchie (S.L.), Rowan (W.B.), 1961 : Effects of water velocities on worm burdens of animals exposed to Schistosoma mansoni cercariae released under laboratory and field conditions. Exp. Parasitol., 11, 323-331.

Richards (C.S.), 1967 : Estivation of Biomphalaria glabrata (Basommatophora, Planorbidae). Associated characteristics and relation to infection with Schistosoma mansoni. Am. J. Trop. Med. Hyg., 16, 797-802.

Rioux (J.A.), Decamps (H.), Lanotte (G.), Combes (C.), Théron (A.), Pointier (J.P.), Seytor (S.), Delattre (P.), Bougerol (C.), 1977 : Ecologie de la Schistosomose en Guadeloupe. Analyse de système épidémiologique. Documents pour un essai de modélisation. Rev. Epidémiol. Santé Pub., 25, 483-519.

Théron (A.), 1979: A differential filtration technique for the measurement of Schistosome cercarian densities in standing water. Bull. Wld. Hlth. Org. (sous presse).

Théron (A.), Pointier (J.P.), Combes (C.), 1977: Recherches sur les facteurs responsables des densités de cercaires de Schistosoma mansoni en Guadeloupe. Ann. Parasitol., Hum. Comp., 52, 421-433.

Théron (A.), Pointier (J.P.), Combes (C.), 1978: Approche écologique de la responsabilité de l'homme et du rat dans le fonctionnement d'un site de transmission à Schistosoma mansoni en Guadeloupe. Ann. Parasitol. Hum. Comp., 53, 223-234.

Webbe (G.), 1966: The effect of water velocities on the infection of animals exposed to Schistosoma mansoni cercariae. Ann. Trop. Med. Parasitol., 60, 78-84. 